PROCEEDINGS OF THE

AMERICAN MATHEMATICAL SOCIETY

Volume 125, Number 7, July 1997, Pages 2127-2132

S 0002-9939(97)03791-X

\title{
ON ORTHOGONALLY EXPONENTIAL AND ORTHOGONALLY ADDITIVE MAPPINGS
}

\author{
JANUSZ BRZDȨK
}

(Communicated by J. Marshall Ash)

\begin{abstract}
Let $E$ be a real inner product space, $(F,+)$ an abelian $\sigma$-bounded topological group, and $K$ a discrete subgroup of $F$. It is proved that (under suitable assumptions on $E$ ) the Christensen and Baire measurable orthogonally additive functions $g: E \rightarrow F / K$ have particular selections. In consequence, descriptions of measurable orthogonally exponential complex functionals on $E$ are obtained.
\end{abstract}

\section{INTRODUCTION}

Assume the following two hypotheses:

$E$ is a real inner product space with $\operatorname{dim} E>1$,

$\left(\mathrm{H}_{2}\right) \quad(F,+)$ is an abelian topological group and $K$ is a discrete subgroup of $F$

(discrete means that there is a neighbourhood $U \subset F$ of 0 with $K \cap U=\{0\}$ ). We study orthogonally additive functions mapping $E$ into the factor group $F / K$, i.e. functions $g$ satisfying the condition

$$
g(x+y)=g(x)+g(y) \quad \text { for orthogonal } x, y \in E .
$$

We show that if such a function is continuous at a point, or Christensen or Baire measurable, then, under suitable assumptions, there are continuous additive functions $a: R \rightarrow F$ and $A: E \rightarrow F$ such that $a\left(\|x\|^{2}\right)+A(x) \in g(x)$ for $x \in E$. In consequence, we obtain analogues, for the Baire and Christensen measurable functions, of the following theorem of K. Baron and J. Rätz.

Theorem A (see [3], p. 15). Assume $\left(\mathrm{H}_{1}\right)$ and $\left(\mathrm{H}_{2}\right)$. Let $F$ be continuously divisible by 2 (i.e. the mapping $x \rightarrow 2 x$ is a homeomorphism of $F$ onto $F$ ) and $f: E \rightarrow F$ be continuous at the origin and satisfying

$$
f(x+y)-f(x)-f(y) \in K \quad \text { for orthogonal } x, y \in E .
$$

Then there are continuous additive functions $a: R \rightarrow F$ and $A: E \rightarrow F$ such that

$$
f(x)-a\left(\|x\|^{2}\right)-A(x) \in K \quad \text { for } x \in E .
$$

Received by the editors September 8, 1995 and, in revised form, February 8, 1996.

1991 Mathematics Subject Classification. Primary 39B52.

Key words and phrases. Baire measurability, Christensen measurability, orthogonal additivity, orthogonally exponential functional. 
We also generalize Theorem A by showing that $f$ can be supposed continuous at any point and that the assumption of continuous divisibility by 2 can be replaced by the following weaker one:

$$
2 x:=x+x \neq 0 \text { for } x \in F \backslash\{0\} .
$$

Finally, we characterize the Baire and Christensen measurable orthogonally exponential functionals $g: E \rightarrow C$, i.e. solutions of the conditional equation

$$
g(x+y)=g(x) g(y) \quad \text { for orthogonal } x, y \in E .
$$

The orthogonally exponential functionals $g: E \rightarrow C$ which are continuous at the origin or measurable on rays (i.e. for every $x \in E$ the function $t \rightarrow g(t x), t \in R$, is Baire or Lebesgue measurable) have been investigated in [1] and [3]. For the information and bibliography concerning the orthogonally additive functions refer e.g. to [10] and [11].

Throughout the paper $N, Z, Q, R$, and $C$ denote, as usual, the sets of positive integers, integers, rationals, reals, and complex numbers, respectively.

Given $F$ and $K$ satisfying $\left(\mathrm{H}_{2}\right)$, in the factor group $F / K$ we take the factor topology, i.e. a set $U \subset F / K$ is open if the set $p^{-1}(U)$ is open in $F$, where $p: F \rightarrow$ $F / K$ is the natural projection. If $F / K$ is endowed with this topology, then it is a topological group and $p$ is open and continuous.

In the sequel $\operatorname{Chr}(X)$ denotes the family of all Christensen measurable subsets of a Polish linear space $X$ which are not Christensen zero sets (for details concerning Christensen measurability refer to [5] and [6]). Analogously, if $X$ is a topological space, $\operatorname{Bai}(X)$ stands for the family of all subsets of $X$ which are of the second category and with the Baire property (see e.g. [8], p. 92, and [9]). Let us recall that a function mapping a topological space $X$ into a topological space $Y$ is Baire measurable provided, for every open set $U \subset Y$, the set $f^{-1}(U)$ has the Baire property in $X$.

\section{THE MAIN THEOREM}

Let us start with the following definition and lemma.

Definition 1. We say that a topological group $(G,+)$ is $\sigma$-bounded provided, for every open neighbourhood $U \subset G$ of 0 , there is a sequence $\left(x_{n}: n \in N\right) \subset G$ with

$$
H=\bigcup\left\{U+x_{n}: n \in N\right\} .
$$

For instance, every topological group $(G,+)$ possessing a dense countable subset is $\sigma$-bounded.

Lemma 1. Let $E$ be a real inner product space, $D \subset E$, and $D_{0}=\left\{\|x\|^{2}: x \in D\right\}$. The following two conditions hold.

(i) If $E$ is a Polish linear space and $D \in \operatorname{Chr}(E)$, then $D_{0}$ contains a subset of positive Lebesgue measure in $R$.

(ii) If $D \in \operatorname{Bai}(E)$, there is $T \in \operatorname{Bai}(R)$ with $T \subset D_{0}$.

Proof. Take $e \in E$ with $\|e\|=1$ and put $Y=\{z \in E: z \perp e\}$. Then $Y$ is a linear subspace of $E$ and $\operatorname{Re} \oplus Y=E$.

First assume that $E$ is a Polish space and $D \in \operatorname{Chr}(E)$. Then $D$ has a universally measurable subset $D_{1}$ which is not a Haar zero set. Let $m$ be the Lebesgue measure 
in $R, r: R \rightarrow E$ be given by $r(c)=c e$ for $c \in R$, and $L_{k}=\{c \in R: k-1 \leq|c|<k\}$ for $k \in N$. Define a Borel measure $u$ on $E$ by the formula

$$
u(T)=\sum_{k=1}^{\infty} 2^{-k}\left[m\left(L_{k}\right)\right]^{-1} m\left(r^{-1}(T) \cap L_{k}\right)
$$

for every Borel set $T \subset E$. It is easily seen that $u$ extended to the family of all universally measurable subsets of $E$ is a probability measure on $E$, which means that there are $b \in R, y \in Y$ with $u\left(D_{1}+b e+y\right)>0$ (cf. [6]). Thus there is a Borel set $D_{2} \subset D_{1}$ with $u\left(D_{2}+b e+y\right)>0$. Hence $m\left(r^{-1}\left(D_{2}+y\right)\right)>0$. Further, we have $D_{3}:=\left\{c^{2}: c e-y \in D\right\} \supset\left\{c^{2}: c \in r^{-1}\left(D_{2}+y\right)\right\}$ and

$$
c^{2}+\|y\|^{2}=c^{2}\|e\|^{2}+\|y\|^{2}=\|c e-y\|^{2} \quad \text { for } c \in R .
$$

Consequently, $D_{3}$ contains a subset of positive Lebesgue measure and $D_{3}+\|y\|^{2} \subset$ $D_{0}$, which implies the statement (i).

Now, suppose $D \in \operatorname{Bai}(E)$. Define a continuous functional $j: E \rightarrow R$ by $j(x)=$ $\langle x, e\rangle$ for $x \in E$, where $\langle$,$\rangle denotes the inner product in E$. Then $j(c e)=c$ for $c \in R$ and $Y=\operatorname{Ker} j$. Let $g: E \rightarrow R \times Y$ and $h: R \times Y \rightarrow E$ be functions given by

$$
\begin{gathered}
g(z)=(j(z), s(z)) \quad \text { for } z \in E, \\
h(c, y)=c e+y \quad \text { for } c \in R, y \in Y,
\end{gathered}
$$

where $s: E \rightarrow Y$ and $s(c e+y)=y$ for $c \in R, y \in Y$. Next, suppose that $Y$ is equipped with the restriction of the inner product from $E$ and $R \times Y$ is endowed with the product topology. Then it is easily seen that $R \times Y$ is a real topological linear space and $g$ and $h$ are continuous. Thus $g$ is a homeomorphism, because $g=h^{-1}$. Hence $g(D) \in \operatorname{Bai}(R \times Y)$. Consequently there is $y \in Y$ such that

$$
D_{y}:=\{c \in R:(c, y) \in g(D)\} \in \operatorname{Bai}(R)
$$

(see [9], p. 57) and therefore $D_{1}:=\left\{c^{2} \in R: c \in D_{y}\right\} \in \operatorname{Bai}(R)$. Further, since (5) is valid and $\|y\|=\|-y\|, D_{1}+\|y\|^{2} \subset D_{0}$. This completes the proof.

Now, we are in a position to formulate and prove the following

Theorem 1. Suppose that hypotheses $\left(\mathrm{H}_{1}\right)-\left(\mathrm{H}_{3}\right)$ are valid and $g: E \rightarrow F / K$ is a function satisfying (1). Further, assume that one of the following three conditions holds:

(i) $E$ is a Polish space, $F$ is $\sigma$-bounded, and $g$ is Christensen measurable;

(ii) $E$ is a Baire space (i.e. it is of the second category), $F$ is $\sigma$-bounded, and $g$ is Baire measurable;

(iii) $g$ is continuous at a point $x_{0} \in E$.

Then there are continuous additive functions $a: R \rightarrow F$ and $A: E \rightarrow F$ such that

$$
a\left(\|x\|^{2}\right)+A(x) \in g(x) \quad \text { for } x \in E .
$$

Proof. Define functions $g_{0}, g_{1}, g_{2}: E \rightarrow F / K$ by $g_{0}(x)=g(-x), g_{1}(x)=g(x)-$ $g(-x)=g(x)-g_{0}(x)$, and $g_{2}(x)=g(x)+g(-x)=g(x)+g_{0}(x)$ for $x \in E$. It is easily seen that $g_{1}$ is odd and $g_{2}$ is even, and they are solutions of (1). Thus, by Theorems 5 and 9 in [10], $g_{1}$ is additive and there is an additive function $h: R \rightarrow F / K$ such that

$$
g_{2}(x)=h\left(\|x\|^{2}\right) \quad \text { for } x \in E .
$$

We will show that $g_{1}$ and $h$ are continuous at the origins in $E$ and $R$, respectively. 
First consider the case (iii) where $g$ is continuous at a point $x_{0}$. Then, in view of the definitions, $g_{1}$ and $g_{2}$ are continuous at $x_{0}$, too. Thus $g_{1}$ is continuous at 0 , because it is additive. Take a neighbourhood $W \subset F / K$ of 0 . There are neighbourhoods $U \subset F / K$ and $V \subset E$ of the respective origins such that $U-U \subset W$ and

$$
g_{2}\left(V+x_{0}\right) \subset U+g_{2}\left(x_{0}\right) .
$$

Put $S=\left\{\|x\|^{2}: x \in V+x_{0}\right\}$. It is easily seen that $\operatorname{int}(S) \neq \varnothing$ in $R$. Hence $S-S$ is a neighbourhood of 0 in $R$. To complete the proof of continuity of $h$ at 0 it suffices to observe that

$$
h(S-S)=h(S)-h(S)=g_{2}\left(V+x_{0}\right)-g_{2}\left(V+x_{0}\right) \subset U-U \subset W .
$$

Now, assume that condition (i) ((ii), respectively) holds. Fix a neighbourhood $W \subset F / K$ of 0 . There are open neighbourhoods $V, U \subset F / K$ of 0 such that $V=-V, V+V \subset U$, and $U-U \subset W$. Furthermore, since $F$ is $\sigma$-bounded, $F / K$ is $\sigma$-bounded, too, and consequently there exists a sequence $\left(x_{n}: n \in N\right) \subset F / K$ such that

$$
F / K=\bigcup\left\{V+x_{n}: n \in N\right\}
$$

Note that

$$
E=g^{-1}(F / K) \cap g_{0}^{-1}(F / K)=\bigcup\left\{g^{-1}\left(V+x_{n}\right) \cap g_{0}^{-1}\left(V+x_{k}\right): n, k \in N\right\} .
$$

Thus there are $n, k \in N$ such that the set

$$
D:=g^{-1}\left(V+x_{n}\right) \cap g_{0}^{-1}\left(V+x_{k}\right)
$$

belongs to $\operatorname{Chr}(E)\left(\operatorname{Bai}(E)\right.$, resp.) and, by Lemma 1 , the set $D_{0}$ contains a subset of positive Lebesgue measure in $R$ (a subset from $\operatorname{Bai}(R)$, resp.). Hence, on account of Theorem 2 in [5] (the Difference Theorem in [8], p. 92, resp.), $0 \in \operatorname{int}(D-D)$ (in $E$ ) and $0 \in \operatorname{int}\left(D_{0}-D_{0}\right)$ (in $R$ ). Since

$$
\begin{aligned}
g_{1}(D-D)= & g_{1}(D)-g_{1}(D) \subset\left(g(D)-g_{0}(D)\right)-\left(g(D)-g_{0}(D)\right) \\
& \subset\left[\left(V+x_{n}\right)-\left(V+x_{k}\right)\right]-\left[\left(V+x_{n}\right)-\left(V+x_{k}\right)\right] \subset U-U \subset W
\end{aligned}
$$

and

$$
\begin{aligned}
h\left(D_{0}-D_{0}\right)= & h\left(D_{0}\right)-h\left(D_{0}\right)=g_{2}(D)-g_{2}(D) \\
& \subset\left(g(D)+g_{0}(D)\right)-\left(g(D)+g_{0}(D)\right) \subset U-U \subset W,
\end{aligned}
$$

this ends the proof of continuity of $g_{1}$ and $h$ at the origins.

It results from Lemma 1 in [4] that there are functions $s_{1}: E \rightarrow F$ and $s_{2}: R \rightarrow F$ continuous at the origins with $s_{1}(x) \in g_{1}(x)$ for $x \in E$ and $s_{2}(c) \in h(c)$ for $c \in R$. Moreover, $s_{1}(x+y)-s_{1}(x)-s_{1}(y) \in K$ for $x, y \in E$ and $s_{2}(c+d)-s_{2}(c)-s_{2}(d) \in K$ for $c, d \in R$, because $g_{1}$ and $h$ are additive. Consequently, in view of Theorem 3 in [2], there are additive and continuous functions $A_{0}: E \rightarrow F$ and $a_{0}: R \rightarrow F$ such that $A_{0}(x) \in g_{1}(x)$ for $x \in E$ and $a_{0}(c) \in h(c)$ for $c \in R$. Let $A: E \rightarrow F$ and $a: R \rightarrow F$ be given by:

$$
\begin{gathered}
A(x)=A_{0}\left(\frac{1}{2} x\right) \quad \text { for } x \in E, \\
a(c)=a_{0}\left(\frac{1}{2} c\right) \quad \text { for } c \in R .
\end{gathered}
$$

Then they are continuous and additive. It remains to show that (6) holds. 
To this end take a function $f: E \rightarrow F$ with $f(x) \in g(x)$ for $x \in E$, which means that $f$ satisfies (2). For every $x \in E$ put $f_{1}(x)=f(x)-f(-x)$ and $f_{2}(x)=$ $f(x)+f(-x)$. Then $f_{i}(x) \in g_{i}(x)$ for $x \in E, i=1,2$, and consequently

$$
\begin{gathered}
f_{1}(x)-A_{0}(x) \in K \quad \text { for } x \in E, \\
f_{2}(x)-a_{0}\left(\|x\|^{2}\right) \in K \quad \text { for } x \in E .
\end{gathered}
$$

Let $k: E \rightarrow K$ be a function defined by the formula:

$k(x)=f_{1}(x)-A_{0}(x)+f_{2}(x)-a_{0}\left(\|x\|^{2}\right)=2 f(x)-A_{0}(x)-a_{0}\left(\|x\|^{2}\right)$ for $x \in E$.

Since, for every $x \in E$,

$$
k(x)+k(-x)=2\left[f(x)+f(-x)-a_{0}\left(\|x\|^{2}\right)\right]=2\left[f_{2}(x)-a_{0}\left(\|x\|^{2}\right)\right] \in 2 K,
$$

according to Theorem 5 in [10], the function $k_{0}: E \rightarrow K / 2 K$, given by $k_{0}(x)=$ $k(x)+2 K$ for $x \in E$, is additive. Thus $k_{0}(x)=2 k_{0}\left(\frac{1}{2} x\right)=0$ for $x \in E$ and therefore $k(E) \subset 2 K$. Whence, for every $x \in E$,

$$
2\left[f(x)-A(x)-a\left(\|x\|^{2}\right)\right]=2 f(x)-A_{0}(x)-a_{0}\left(\|x\|^{2}\right)=k(x) \in 2 K,
$$

which jointly with $\left(\mathrm{H}_{3}\right)$ yields $(6)$. This ends the proof.

\section{Applications}

Now, we present two theorems which result from Theorem 1. The first one is a generalization of Theorem 1 in [3] and contains a result concerning stability, of Hyers-Ulam type (see e.g. [7]), for orthogonally additive mappings; the second characterizes orthogonally exponential functionals.

Theorem 2. Suppose $\left(\mathrm{H}_{1}\right)-\left(\mathrm{H}_{3}\right)$. Let $f: E \rightarrow F$ be a function satisfying (2). If one of conditions (i)-(iii) of Theorem 1 is valid with $g=f$, then there exist continuous additive functions $a: R \rightarrow F$ and $A: E \rightarrow F$ such that (3) holds.

Proof. Put $g=p \circ f$, where $p: F \rightarrow F / K$ is the natural projection. Then one of conditions (i)-(iii) of Theorem 1 is satisfied. Thus Theorem 1 implies the assertion.

Theorem 3. Let $E$ be a real inner product space with $\operatorname{dim} E>1$ and $h: E \rightarrow C$ be a function satisfying (4). Suppose that one of the following three conditions is valid:

(i) $E$ is a Polish space and $h$ is Christensen measurable;

(ii) $E$ is a Baire space and $h$ is Baire measurable;

(iii) $h$ is continuous at a point.

Then either $h(x)=0$ for $x \in E$ or

$$
h(x)= \begin{cases}0 & \text { if } x \in E \backslash\{0\}, \\ 1 & \text { if } x=0\end{cases}
$$

or there are $c \in C$ and a continuous $R$-linear functional $A: E \rightarrow C$ such that

$$
h(x)=\exp \left(c\|x\|^{2}+A(x)\right) \quad \text { for } x \in E .
$$


Proof. Suppose that $h(x) \neq 0$ for some $x \in E \backslash\{0\}$. Then, according to Proposition 3 in [1], $0 \notin h(E)$. Let $S=\{z \in C:|z|=1\}$ and $h_{0}: E \rightarrow S, f: E \rightarrow R$, $g: E \rightarrow R / Z, T: S \rightarrow R / Z$ be functions given by $f(x)=\log |h(x)|$ for $x \in E$,

$$
\begin{gathered}
h_{0}(x)=\frac{h(x)}{|h(x)|} \quad \text { for } x \in E, \\
T(\exp 2 \pi i t)=t+Z \quad \text { for } t \in[0,1),
\end{gathered}
$$

and $g=T \circ h_{0}$. It is easily seen that $g$ and $f$ satisfy the assumptions of Theorems 1 and 2 , respectively, with $F=R$ and $K=Z$, and, moreover, $f$ satisfies (1), i.e. (2) with $K=\{0\}$. Thus there are $c_{1}, c_{2} \in R$ and continuous linear functionals $A_{1}, A_{2}: E \rightarrow R$ with

$$
\begin{aligned}
& f(x)=c_{1}\|x\|^{2}+A_{1}(x) \quad \text { for } x \in E, \\
& c_{2}\|x\|^{2}+A_{2}(x) \in g(x) \quad \text { for } x \in E .
\end{aligned}
$$

Since $h(x)=h_{0}(x) \exp (f(x))$ for $x \in E$, setting $c=c_{1}+2 \pi i c_{2}$ and $A=A_{1}+2 \pi i A_{2}$ we obtain the statement.

Remark. It results from Remark in [3] (on page 15) that the regularity assumptions made in Theorems 1-3 are essential.

\section{REFERENCES}

1. K. Baron, F. Halter-Koch, and P. Volkmann, On orthogonally exponential functions, Arch. Math. (Basel) 64 (1995), 410-414. CMP 95:10

2. K. Baron and PL. Kannappan, On the Pexider difference, Fund. Math. 134 (1990), 247-254. MR 92a:39013

3. K. Baron and J. Rätz, Orthogonality and additivity modulo a subgroup, Aequationes Math. 46 (1993), 11-18. MR 94j:39016

4. J. Brzdęk, On the Cauchy difference, Glasnik Mat. 27(47) (1992), 263-269. MR 95a:39021

5. J. P. R. Christensen, On sets of Haar measure zero in abelian Polish groups, Israel J. Math. 13 (1972), 255-260. MR 48:4637

6. P. Fischer and Z. Slodkowski, Christensen zero sets and measurable convex functions, Proc. Amer. Math. Soc. 79 (1980), 449-453. MR 81d:28013

7. D. H. Hyers and Th. M. Rassias, Approximate homomorphisms, Aequationes Math. 44 (1992), 125-153. MR 93i:39007

8. J. L. Kelley, I. Namioka, et al., Linear topological spaces, Springer-Verlag, 1976. MR 52:14890

9. J. C. Oxtoby, Measure and category, Graduate Texts in Mathematics, Springer-Verlag, 1971. MR 52:14213

10. J. Rätz, On orthogonally additive mappings, Aequationes Math. 28 (1985), 35-49. MR 87b:39012

11. K. Sundaresan, Orthogonality and nonlinear functionals on Banach spaces, Proc. Amer. Math. Soc. 34 (1972), 187-190. MR 45:925

Department of Mathematics, Pedagogical University, Rejtana 16 A, 35-310 Rzeszow, POLAND 